\title{
Composição da avifauna em duas matas ciliares na bacia do rio Jacaré-Pepira, São Paulo, Brasil
}

\author{
Maria Elisa de Castro Almeida ${ }^{1}$ \\ Jacques M.E. Vielliard ${ }^{2}$ \\ Manoel Martins Dias ${ }^{1}$
}

\begin{abstract}
Bird community composition of two riparian forests at Jacaré-Pepira river, São Paulo, Brazil. A quali-quantitative survey was carried out in two riparian forests fragments (approximately 40 ha each) at Jacaré-Pepira river. Our intention was to characterize its bird community concerning richness, abundance and species occurrence in these areas. The qualitative survey showed 130 species at Santa Elisa (Brotas, São Paulo) and 151 at Morro Chato (Dourado, São Paulo), whereas the quantitative survey revealed the presence of 69 and 75 species at Santa Elisa and Morro Chato, respectively. The small size and the isolation might be responsible for the low number of species found. Observing the abundance index values (IPA) we realize that there are a few number of species with a high IPA on the one hand, while on the other there is a large number of species with intermediate and low IPA rates. A high detection coefficient (vocalization), low predatory rates and competition might have contributed for the higher abundance values found among these species. Furthermore, we have also registered species which are abundant in a fragment, but absent in the other, which might be explained by initial exclusion or local extinction. In spite of the riparian forests being protected by law, its clearing process has not stopped yet. Therefore, the study of this bird community is of uppermost importance for the elaboration of both conservation and management projects regarding these areas.
\end{abstract}

KEY WORDS. Bird community, unlimited distance point counts, riparian forest

$\mathrm{O}$ presente trabalho teve como objetivo caracterizar (qualitativamente e quantitativamente) a estrutura das comunidades de aves em relação a riqueza, abundância e freqüência de ocorrência das espécies em dois remanescentes de mata ciliar na bacia do rio Jacaré-Pepira, estado de São Paulo, fornecendo elementos de comparação para futuros trabalhos, contribuindo para uma melhor caracterização da avifauna das matas ciliares e para a conservação da biodiversidade nestas áreas.

A avifauna em mata ciliar é pouco estudada e faltam levantamentos quantificados de comunidades de aves para analisar as correlações entre a estrutura da avifauna e a composição florística e espacial da vegetação nas matas ciliares. Estas informações são indispensáveis para caracterizar e monitorar esses ecossistemas, preservando a biodiversidade que eles sustentam (SILVA \& VIELLIARD no prelo). Apesar de já existirem alguns levantamentos quantitativos da avifauna no Brasil (ALEIXO \& ViELLIARD 1995; ViELLIARD no prelo), o único levantamento quantificado de avifauna em mata ciliar disponível até o momento foi realizado em Lençóis Paulista, São Paulo por Vielliard \& SilVA (1990).

1) Departamento de Ecologia e Biologia Evolutiva, Universidade Federal de São Carlos. Caixa Postal 676, 13565-905 São Carlos, São Paulo, Brasil.

2) Departamento de Zoologia, Universidade Estadual de Campinas. Caixa Postal 6109, 13083-970 Campinas, São Paulo, Brasil. 


\section{MATERIAL E MÉTODOS}

\section{Área de estudo}

A bacia do rio Jacaré-Pepira, localizada no estado de São Paulo, pertence à bacia do rio Paraná e ocupa uma área de $2612 \mathrm{~km}^{2}$. A paisagem é essencialmente agrícola e a vegetação natural está reduzida a fragmentos de mata e estreitos corredores de matas ribeirinhas. A bacia situa-se na zona de influência de três tipos de formações vegetacionais: cerrados, matas mesófilas de altitude e matas mesófilas semidecíduas.

Foram estudados dois fragmentos de mata ciliar localizados na bacia do rio Jacaré-Pepira, um no município de Brotas ( $\left.48^{\circ} 08^{\prime} \mathrm{W} ; 22^{\circ} 17^{\prime} \mathrm{S}\right)$ e outro em Dourado $\left(21^{\circ} 22^{\prime} \mathrm{S} ; 49^{\circ} 41^{\prime} \mathrm{W}\right)$. O fragmento de mata da fazenda Santa Elisa (Brotas) possui $1250 \mathrm{~m}$ de comprimento e área de 37 ha. Áreas de pastagem, um pequeno brejo e a sede da fazenda compõem a paisagem circundante. $\mathrm{O}$ fragmento da fazenda Morro Chato (Dourado) possui $1000 \mathrm{~m}$ de comprimento e 27 ha. A paisagem da fazenda é diversificada, com pomares, pastos, áreas de cultivo e um banhado.

O clima da região é do tipo Cwa, apresentando inverno seco. A precipitação média anual varia entre 1100 e $1400 \mathrm{~mm}$, sendo julho o mês mais seco (30 mm) e dezembro o mês mais chuvoso $(250 \mathrm{~mm})$. A temperatura média anual varia entre $21^{\circ} \mathrm{C}$ e $23^{\circ} \mathrm{C}$. O mês com temperatura média mais alta é fevereiro $\left(25,1^{\circ} \mathrm{C}\right)$ e o de temperatura média mais baixa é julho $\left(18,7^{\circ} \mathrm{C}\right)$.

\section{Métodos de amostragem da avifauna}

Foram realizados dois tipos de levantamentos: qualitativo e quantitativo, através do Método de Amostragem por Pontos (BLONDEL et al. 1970; ViELLiARD \& Silva 1990).

Durante os meses de fevereiro de 1994 a setembro de 1995 foram realizadas 25 visitas em cada área estudada, com uma média de 7,5 horas por visita, totalizando 175 horas de observação em cada fragmento estudado. Devido à grande atividade das aves em setembro, realizou-se um maior número de visitas neste mês, a fím de amostrar um maior número de espécies para o levantamento qualitativo (Tab. I).

Tabela I. Meses e datas das visitas.

\begin{tabular}{lcc}
\hline \multicolumn{1}{c}{ Meses } & 1994 (dias) & 1995 (dias) \\
\hline Janeiro & - & $16,17,27$ e28 \\
Fevereiro & 24 e 25 & - \\
Março & 21 e 22 & - \\
Abril & 15 e 16 & - \\
Maio & 25 e 26 & - \\
Junho & 17 e 18 & - \\
Julho & 19 e 20 & 2 a 11 \\
Agosto & 29 e 30 & - \\
Setembro & 24 a 30 & - \\
Outubro & 1 a 3,27 e 28 & - \\
Novembro & $13,14,23$ e 24 & - \\
Dezembro & 16 e 17 & \\
\hline
\end{tabular}




\section{Levantamento qualitativo}

O levantamento qualitativo foi realizado em caminhadas ao longo de uma trilha de $1000 \mathrm{~m}$ de comprimento, paralela ao rio. Os ambientes adjacentes às matas, como banhado, lagoa, pastagens e áreas de cultivo também foram considerados. $\mathrm{O}$ levantamento iniciava-se às 6:00 $\mathrm{h}$. Durante o percurso foram registrados: identificação da espécie, ambiente (mata, banhado ou áreas adjacentes), tipo de contato estabelecido (visual e/ou auditivo), vocalizações e número de indivíduos.

\section{Levantamento quantitativo}

Para este levantamento foram utilizadas as mesmas trilhas do levantamento qualitativo. Foram alocados cinco pontos ao longo das trilhas, distantes $200 \mathrm{~m}$ entre $\mathrm{si}$, que foram amostrados entre 6:00 e 10:30 h. A ordem de amostragem foi estabelecida através de sorteio e as sessões de observações duravam 20 minutos em cada ponto, conforme metodologia estabelecida por VIELLIARD \& SILVA (1990). Todas as espécies vistas e ouvidas foram anotadas.

Devido ao fato da mata ciliar ser muito estreita, todas as espécies de aves foram consideradas, sem distinção entre espécies de interior e borda. O levantamento quantitativo restringiu-se à área de mata e as espécies registradas neste levantamento também foram anotadas na listagem qualitativa.

\section{RESULTADOS E DISCUSSÃO}

\section{Levantamento qualitativo}

\section{Número de espécies}

Registrou-se um total de 130 espécies em Santa Elisa (SE) e 151 em Morro Chato (MC). Em Santa Elisa 74\% (96 espécies) habitam a mata e 26\% (34 espécies) os ambientes adjacentes (pastagens e brejos). Em Morro Chato 68\% (102 espécies) habitam a mata e $32 \%$ (49 espécies) os ambientes adjacentes.

O número de espécies encontradas no levantamento qualitativo é baixo quando comparado com o estudo realizado por Vielliard \& Silva (1990) com 180 espécies. Porém este número pode ser considerado alto quando comparado ao resultado obtido por Aleixo \& Vielliard (1995), 134 espécies em 251 ha.

\section{Freqüência de ocorrência}

A freqüência de ocorrência relaciona a proporção dos dias em que a espécie foi encontrada com o número total de dias de levantamento, permitindo concluir se uma espécie é regularmente encontrada ou não (VIELLIARD \& Silva 1990). A freqüência de ocorrência das espécies foi dividida em intervalos conforme mostra a tabela II.

A freqüência de ocorrência acima de $75 \%$ engloba espécies residentes (aquelas encontradas em mais de seis visitas - freqüência de ocorrência maior que $25 \%$ ) abundantes que representam aproximadamente $7 \%$ das espécies encontradas em SE e MC como Automolus leucophthalmus (Wied, 1821), Herpsilochmus pileatus (Pelzeln, 1868), Antilophia galeata (Lichtenstein, 1823), Pitangus sulphuratus (Linnaeus, 1766), Cyclarhis gujanensis (Gmelin, 1789), Basileuterus flaveolus (Baird, 1865) e Basileuterus culicivorus (Lichtenstein, 1830). 
Tabela II. Porcentagem de espécies por classes de freqüência de ocorrência.

\begin{tabular}{lcc}
\hline Freqüência de ocorrência (\%) & Santa Elisa (\%) & Morro Chato (\%) \\
\hline$<25$ & 65,4 & 61,8 \\
$25-49$ & 19,2 & 13,8 \\
$50-74$ & 9,2 & 15,8 \\
$75-99$ & 7,6 & 7,9 \\
100 & 0,0 & 1 espécie \\
\hline
\end{tabular}

A maior parte da avifauna, $65 \%$ em SE e $62 \%$ em MC, é constituída por espécies com freqüência de ocorrência inferior a $25 \%$, que apareceram em menos de sete do total de 25 visitas. A baixa freqüência de ocorrência e o registro único de algumas espécies podem ser explicados pelo aparecimento de espécies que permanecem poucos dias no local (vagantes), espécies que habitam outros ambientes e ocasionalmente exploram algum recurso da mata (ocasionais), ou espécies migratórias como Dendrocygna viduata (Linnaeus, 1766), Porphyrula martinica (Linnaeus, 1766) e Elaenia spectabilis Pelzeln, 1868 (Tab. III). Outros fatores que explicam o registro único de algumas espécies, segundo ALEIXO \& VIELLIARD (1995), são vocalização pouco conspícua e/ou baixa densidade populacional na área. Para os autores, somente uma análise ecológica de cada espécie explicaria melhor os baixos valores de freqüência de ocorrência encontrados.

\section{Levantamento quantitativo}

Número de espécies

Foram registradas 69 espécies em Santa Elisa e 75 em Morro Chato, num total de 110 amostras realizadas em cada área. Em SE o número de espécies encontradas em cada visita ( 5 amostras por visita) variou de 13 (dezembro) a 21 (janeiro); em MC variou de 11 (julho) a 27 (setembro) (Fig. 1).

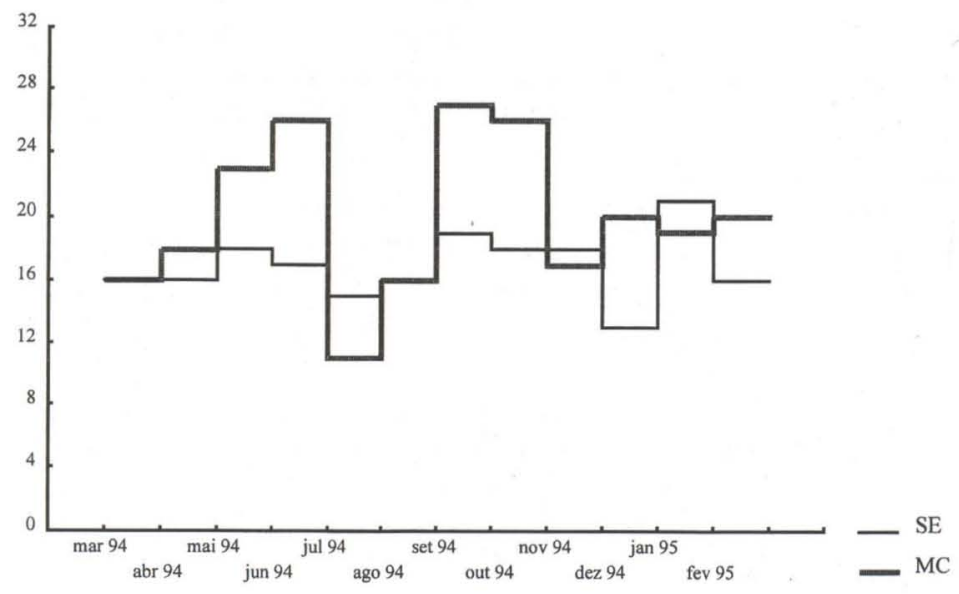

Fig. 1. Variação anual do número de espécies encontradas por visita. 
Tabela III. Lista sistemática da avifauna nas matas de Santa Elisa (SE) e Morro Chato (MC), com indicação do hábitat, freqüência de ocorrência, indice pontual de abundância e status (seguindo nomenclatura de MEYER DE SCHAUENSEE 1966, 1970). (HAB) Habitat; (M) mata; (P) áreas de pastagens; $(B)$ banhado; ( $F O)$ freqüência de ocorrência ( $F O=4 \%$ - espécie encontrada em apenas uma visita); (IPA) índice pontual de abundância (um contato=0,009); (status) relação entre a espécie e as áreas estudadas; (res) residentes, espécies encontradas em seis visitas ou mais; (oc) ocasionais, espécies habitantes de outros ambientes que ocasionalmente exploram algum recurso da mata; (vag) vagantes, espécies de ocorrência irregular na mata ou que foram encontradas apenas uma única vez; (mig) migratórias, espécies migratórias.

\begin{tabular}{|c|c|c|c|c|c|c|}
\hline \multirow{2}{*}{ Espécies } & \multirow{2}{*}{ HAB } & \multicolumn{2}{|c|}{ FO $(\%)$} & \multicolumn{2}{|c|}{ IPA } & \multirow{2}{*}{ Status } \\
\hline & & SE & MC & SE & MC & \\
\hline \multicolumn{7}{|l|}{ Tinamidae } \\
\hline Crypturellus tataupa (Temminck, 1815) & M & 4 & 4 & - & - & vag \\
\hline Nothura maculosa (Temminck, 1815) & $P$ & - & 4 & - & 0,009 & oc \\
\hline \multicolumn{7}{|l|}{ Ardeidae } \\
\hline Ardea cocoi Linnaeus, 1766 & B & - & 24 & - & - & oc \\
\hline Casmerodius albus (Linnaeus, 1758) & $\mathrm{B}, \mathrm{P}$ & 8 & 16 & - & - & $\mathrm{oc}$ \\
\hline Egretta thula (Molina, 1782) & B & 4 & 4 & - & - & vag \\
\hline Syrigma sibilatrix (Temminck, 1824) & B & 32 & 20 & - & - & oc \\
\hline \multicolumn{7}{|l|}{ Threskiornithidae } \\
\hline Mesembrinibis cayennensis (Gmelin, 1789) & B & 8 & 4 & - & - & mig \\
\hline Plegadis chihi (Vieillot, 1817) & B & - & 4 & - & - & mig \\
\hline \multicolumn{7}{|l|}{ Anatidae } \\
\hline Dendrocygna viduata (Linnaeus, 1766) & B & - & 4 & - & - & $o c$ \\
\hline Amazonetta brasiliensis (Gmelin, 1789) & B & 4 & 4 & - & - & vag \\
\hline Cairina moschata (Linnaeus, 1758) & B & - & 16 & - & - & vag \\
\hline \multicolumn{7}{|l|}{ Cathartidae } \\
\hline Coragyps atratus (Bechstein, 1793) & M & 88 & 24 & 0,018 & 0,009 & res \\
\hline \multicolumn{7}{|l|}{ Accipitridae } \\
\hline Elanus leucurus (Vieillot, 1818) & $\mathrm{P}$ & - & 4 & - & - & vag \\
\hline Buteo magnirostris (Gmelin, 1788) & $M, P$ & 64 & 68 & - & - & res \\
\hline Busarellus nigricollis (Latham, 1790) & B & - & 12 & - & - & oc \\
\hline Heterospizias meridionalis (Latham, 1790) & $\mathrm{M}, \mathrm{P}$ & - & 8 & - & - & vag \\
\hline \multicolumn{7}{|l|}{ Falconidae } \\
\hline Herpetotheres cachinnans (Linnaeus, 1758) & $M, P$ & 16 & 4 & 0,018 & - & $O C$ \\
\hline Milvago chimachima (Vieillot, 1816) & $\mathrm{P}$ & 8 & 16 & 0,009 & - & oc \\
\hline Polyborus plancus (Miller, 1777) & $\mathrm{P}$ & - & 8 & - & - & $O C$ \\
\hline Falco sparverius Linnaeus, 1758 & $P$ & 20 & 16 & - & - & oc \\
\hline \multicolumn{7}{|l|}{ Rallidae } \\
\hline Rallus nigricans Vieillot, 1819 & B & - & 4 & - & - & vag \\
\hline Aramides cajanea (Müller, 1776) & B & 20 & 16 & - & - & $o c$ \\
\hline Laterallus leucopyrrhus (Vieillot, 1819) & B & 4 & - & - & - & vag \\
\hline Gallinula chloropus (Linnaeus, 1758) & $\mathrm{B}$ & 4 & 68 & - & - & mig \\
\hline Porphyrula martinica (Linnaeus, 1766) & B & 4 & 8 & - & - & $\mathrm{oc}$ \\
\hline \multicolumn{7}{|l|}{ Cariamidae } \\
\hline Cariama cristata (Linnaeus, 1766) & $\mathrm{P}$ & 60 & 76 & - & - & oc \\
\hline \multicolumn{7}{|l|}{ Jacanidae } \\
\hline Jacana jacana (Linnaeus, 1766) & B & - & 68 & - & - & mig \\
\hline \multicolumn{7}{|l|}{ Charadriidae } \\
\hline Vanellus chilensis (Molina, 1782) & $B, P$ & 72 & 64 & - & - & res \\
\hline \multicolumn{7}{|l|}{ Columbidae } \\
\hline Columba picazuro Temminck, 1813 & M & 24 & 48 & 0,018 & 0,018 & mig \\
\hline Columba cayennensis Bonnaterre, 1792 & M & 56 & 68 & 0,173 & 0,291 & res \\
\hline Columbina talpacoti (Temminck, 1811) & $\mathrm{P}$ & 36 & 48 & 0,109 & 0,127 & res \\
\hline Scardafella squammata (Lesson, 1831) & $M, P$ & 40 & 16 & 0,073 & 0,064 & res \\
\hline Leptotila verreauxi (Bonaparte, 1855) & M & 56 & 68 & 0,209 & 0,327 & res \\
\hline Leptotila rufaxilla (Richard \& Bernard, 1792) & M & 36 & 44 & 0,155 & 0,327 & res \\
\hline \multicolumn{7}{|l|}{ Psittacidae } \\
\hline Aratinga leucophthalmus (Müller, 1776) & M & 24 & 56 & 0,018 & 0,036 & res \\
\hline & & & & & & ontinu \\
\hline
\end{tabular}


Tabela III. Continuação.

\begin{tabular}{|c|c|c|c|c|c|c|}
\hline \multirow{2}{*}{ Espécies } & \multirow{2}{*}{$\mathrm{HAB}$} & \multicolumn{2}{|c|}{$\mathrm{FO}(\%)$} & \multicolumn{2}{|c|}{ IPA } & \multirow{2}{*}{ Status } \\
\hline & & SE & MC & SE & MC & \\
\hline Forpus xanthopterygius (Spix, 1824) & $M, P$ & 12 & 8 & 0,009 & - & oc \\
\hline Brotogeris versicolurus (Maller, 1776) & $\mathrm{M}, \mathrm{P}$ & 48 & 52 & 0,073 & 0,055 & res \\
\hline Pionus maximiliani (Kuhl, 1820) & $M, P$ & 4 & 4 & - & - & vag \\
\hline Amazona aestiva (Linnaeus, 1758) & $M, P$ & 32 & 76 & 0,027 & 0,036 & res \\
\hline \multicolumn{7}{|l|}{ Cuculidae } \\
\hline Piaya cayana (Linnaeus, 1766) & M & 68 & 40 & 0,118 & 0,091 & res \\
\hline Crotophaga ani Linnaeus, 1758 & $\mathrm{P}$ & 16 & 72 & - & - & oc \\
\hline Guira guira (Gmelin, 1788) & $\mathrm{P}$ & 12 & 52 & - & - & oc \\
\hline Tapera naevia (Linnaeus, 1766) & M & 28 & 56 & 0,073 & 0,145 & oc \\
\hline \multicolumn{7}{|l|}{ Strigidae } \\
\hline Speotyto cunicularia (Molina, 1782) & $\mathrm{P}$ & 8 & 40 & - & - & oc \\
\hline \multicolumn{7}{|l|}{ Caprimulgidae } \\
\hline Nyctidromus albicollis (Gmelin, 1789) & M & 4 & 48 & - & 0,027 & res \\
\hline Caprimulgus rufus Boddaert, 1783 & M & - & 4 & - & - & res \\
\hline Hydropsalis brasiliana (Gmelin, 1789) & M & - & 4 & - & - & res \\
\hline \multicolumn{7}{|l|}{ Trochilidae } \\
\hline Phaethornis pretrei (Lesson \& Delattre, 1839) & $M, B$ & 12 & 12 & - & - & vag \\
\hline Eupetomena macroura (Gmelin, 1788) & $M_{1} B$ & 4 & 16 & - & - & vag \\
\hline Melanotrochilus fuscus (Vieillot, 1817) & M & 4 & 16 & - & 0,045 & mig \\
\hline $\begin{array}{l}\text { Chlorostilbon aureoventris (d'Orbigny \& } \\
\text { Lafresnaye, 1838) }\end{array}$ & $M$ & 4 & 12 & - & - & vag \\
\hline Thalurania glaucopis (Gmelin, 1788) & M & 4 & - & - & - & vag \\
\hline Hylocharis chrysura (Shaw, 1812) & M & - & 4 & - & - & vag \\
\hline Leucochloris albicollis (Vieillot, 1818) & M & 4 & 4 & - & - & vag \\
\hline Amazilia versicolor (Vieillot, 1818) & M & - & 4 & - & - & vag \\
\hline Amazilia fimbriata (Gmelin, 1788) & M & 4 & 12 & 0,009 & 0,009 & vag \\
\hline Amazilia lactea (Lesson, 1829) & M & - & 8 & - & - & vag \\
\hline \multicolumn{7}{|l|}{ Trogonidae } \\
\hline Trogon surrucura Vieillot, 1817 & M & 8 & 12 & 0,018 & 0,055 & vag \\
\hline \multicolumn{7}{|l|}{ Alcedinidae } \\
\hline Ceryle torquata (Linnaeus, 1766) & B & 16 & 20 & - & - & $o c$ \\
\hline Chloroceryle amazona (Latham, 1790) & $\mathrm{B}$ & 4 & 4 & - & - & oc \\
\hline Chloroceryle americana (Gmelin, 1788) & B & - & 16 & - & - & oc \\
\hline \multicolumn{7}{|l|}{ Momotidae } \\
\hline Baryphthengus ruficapillus (Vieillot, 1818) & M & 8 & - & 0,018 & - & vag \\
\hline \multicolumn{7}{|l|}{ Galbulidae } \\
\hline \multicolumn{7}{|l|}{ Bucconidae } \\
\hline Malacoptila striata (Spix, 1822) & $M$ & 4 & 24 & - & 0,027 & res \\
\hline \multicolumn{7}{|l|}{ Ramphastidae } \\
\hline Ramphastos toco Müller, 1776 & $M, P$ & 8 & 36 & 0,027 & 0,009 & res \\
\hline \multicolumn{7}{|l|}{ Picidae } \\
\hline Picumnus minutissimus (Pallas, 1782) & M & 8 & 16 & - & - & vag \\
\hline Colaptes campestris (Vieillot, 1818) & $\mathrm{P}$ & 36 & 60 & 0,018 & 0,027 & oc \\
\hline Chrysoptilus melanochloros (Gmelin, 1788) & $M, P$ & - & 12 & - & 0,027 & $o c$ \\
\hline Dryocopus lineatus (Linnaeus, 1766) & M & 36 & 12 & 0,100 & 0,018 & res \\
\hline Leuconerpes candidus (Otto, 1796) & $M, P$ & 28 & 24 & 0,055 & - & oc \\
\hline Veniliornis passerinus (Linnaeus, 1766) & M & 40 & 20 & 0,073 & 0,027 & res \\
\hline Phloeoceastes robustus (Lichtenstein, 1819) & M & 8 & 8 & 0,018 & 0,009 & vag \\
\hline Dendrocolaptidae & & & & & & \\
\hline Sittasomus griseicapillus (Vieillot, 1818) & M & 4 & 28 & 0,009 & 0,082 & res \\
\hline Xiphocolaptes albicollis (Vieillot, 1818) & M & - & 8 & - & 0,018 & vag \\
\hline Dendrocolaptes platyrostris Spix, 1824 & M & - & 4 & - & - & vag \\
\hline Lepidocolaptes angustirostris (Vieillot, 1818) & $\mathrm{P}$ & 8 & 28 & 0,009 & 0,018 & $o c$ \\
\hline Furnariidae & & & & & & \\
\hline Furnarius rufus (Gmelin, 1788) & $P$ & - & 12 & - & - & oc \\
\hline Synallaxis ruficapilla Vieillot, 1819 & M & 80 & - & 0,291 & - & res \\
\hline Synallaxis frontalis Pelzeln, 1859 & M & 44 & 16 & - & 0,009 & res \\
\hline Synallaxis spixi Sclater, 1856 & M & 4 & - & - & - & vag \\
\hline
\end{tabular}


Tabela III. Continuação.

\begin{tabular}{|c|c|c|c|c|c|c|}
\hline \multirow{2}{*}{ Espécies } & \multirow{2}{*}{ HAB } & \multicolumn{2}{|c|}{$\mathrm{FO}(\%)$} & \multicolumn{2}{|c|}{ IPA } & \multirow{2}{*}{ Status } \\
\hline & & SE & MC & SE & MC & \\
\hline Certhiaxis cinnamomea (Gmelin, 1788) & $\mathrm{B}$ & 8 & 56 & - & - & OC \\
\hline Automolus leucophthalmus (Wied, 1821) & M & 80 & 76 & 0,445 & 0,255 & res \\
\hline Xenops rutilans Temminck, 1821 & M & 4 & - & - & - & vag \\
\hline \multicolumn{7}{|l|}{ Formicariidae } \\
\hline Taraba major (Vieillot, 1816) & M & 48 & 28 & 0,082 & 0,036 & res \\
\hline Thamnophilus doliatus (Linnaeus, 1764) & M & 56 & 92 & 0,045 & 0,509 & res \\
\hline Thamnophilus punctatus (Shaw, 1809) & M & 8 & 16 & 0,018 & 0,055 & res \\
\hline Thamnophilus caerulescens Vieillot, 1816 & M & 84 & 68 & 0,282 & 0,136 & res \\
\hline Dysithamnus mentalis (Temminck, 1823) & M & 64 & 60 & 0,145 & 0,209 & res \\
\hline Herpsilochmus pileatus (Pelzeln, 1868) & M & 80 & 88 & 0,427 & 0,591 & res \\
\hline Drymophila ferruginea (Temminck, 1822) & M & - & 4 & - & - & vag \\
\hline Pyriglena leucoptera (Vieillot, 1818) & M & 4 & 4 & - & 0,009 & vag \\
\hline Conopophaga lineata (Wied, 1831) & M & 72 & 16 & 0,200 & 0,027 & res \\
\hline \multicolumn{7}{|l|}{ Cotingidae } \\
\hline Pachyramphus polychopterus (Vieillot, 1818) & M & 4 & 8 & 0,009 & 0,009 & mig \\
\hline Platypsaris rufus (Vieillot, 1816) & M & - & 24 & - & - & res \\
\hline Tityra cayana (Linnaeus, 1766) & M & - & 4 & - & 0,009 & vag \\
\hline \multicolumn{7}{|l|}{ Pipridae } \\
\hline Antilophia galeata (Lichtenstein, 1823) & $M, B$ & 80 & 72 & 0,436 & 0,345 & res \\
\hline Chiroxiphia caudata (Shaw \& Nodder, 1793) & M & 4 & - & - & - & vag \\
\hline Neopelma aurifrons (Wied, 1831) & M & 12 & - & 0,009 & - & vag \\
\hline Schiffornis virescens (Lafresnaye, 1824) & $M, P$ & 16 & 4 & 0,027 & 0,009 & vag \\
\hline \multicolumn{7}{|l|}{ Tyrannidae } \\
\hline Xolmis velata (Lichtenstein, 1823) & $P$ & 4 & 24 & - & - & mig \\
\hline Colonia colonus (Vieillot, 1818) & B & 16 & 8 & - & - & mig \\
\hline Gubernetes yetapa (Vieillot, 1818) & B & - & 4 & - & - & mig \\
\hline Arundinicola leucocephala (Linnaeus, 1764) & B & 4 & 28 & - & - & oc \\
\hline Machetornis rixosus (Vieillot, 1819) & $M, P$ & 4 & 16 & - & - & mig \\
\hline Muscivora tyrannus (Linnaeus, 1766) & $M, P$ & 4 & 8 & - & - & mig \\
\hline Tyrannus melancholicus Vieillot, 1819 & $\mathrm{M}, \mathrm{B}$ & 28 & 52 & 0,009 & 0,055 & res \\
\hline Megarynchus pitangua (Linnaeus, 1766) & M & 24 & 60 & 0,045 & 0,118 & res \\
\hline Myiodynastes maculatus (Müller, 1776) & M & 12 & 12 & 0,027 & 0,018 & mig \\
\hline Myiozetetes cayanensis (Linnaeus, 1766) & $M, B$ & 8 & 60 & - & 0,082 & $\mathrm{mig}$ \\
\hline Myiozetetes similis (Spix, 1825) & M & 20 & 32 & 0,045 & 0,182 & $\mathrm{mig}$ \\
\hline Pitangus sulphuratus (Linnaeus, 1766) & $M, P$ & 72 & 100 & 0,191 & 1,082 & res \\
\hline Myiarchus ferox (Gmelin, 1789) & M & 16 & 44 & 0,018 & 0,127 & res \\
\hline Myiarchus tyrannulus (Müller, 1776) & M & 8 & 20 & - & 0,045 & mig \\
\hline Empidonax euleri (Cabanis, 1868) & M & 32 & 28 & 0,064 & 0,036 & res \\
\hline Cnemotriccus fuscatus (Wied, 1831) & M & 44 & 60 & 0,064 & 0,264 & res \\
\hline Platyrinchus mystaceus Vieillot, 1818 & M & 36 & 20 & 0,036 & 0,009 & res \\
\hline Todirostrum poliocephalum (Wied, 1831) & M & 32 & - & 0,018 & - & res \\
\hline Myiornis auricularis (Vieillot, 1818) & M & - & 8 & - & - & vag \\
\hline Elaenia flavogaster (Thunberg, 1822) & $\mathrm{M}, \mathrm{P}$ & 12 & 40 & 0,018 & 0,109 & res \\
\hline Elaenia spectabilis Pelzeln, 1868 & $M, P$ & - & 4 & - & 0,009 & vag \\
\hline Myiopagis vindicata (Vieillot, 1817) & M & 8 & - & 0,009 & - & mig \\
\hline Camptostoma obsoletum (Temminck, 1824) & $M, P$ & 32 & 52 & 0,082 & 0,145 & res \\
\hline Corythopis delalandi (Lesson, 1831) & M & - & 88 & - & 0,318 & res \\
\hline \multicolumn{7}{|l|}{ Hirundinidae } \\
\hline Notiochelidon cyanoleuca (Vieillot, 1817) & $\mathrm{P}$ & 4 & 8 & - & - & mig \\
\hline Stelgidopteryx ruficollis (Vieillot, 1817) & P. B & - & 12 & - & - & mig \\
\hline \multicolumn{7}{|l|}{ Corvidae } \\
\hline Cyanocorax cristatellus (Temminck, 1823) & $\mathrm{P}$ & 4 & 12 & - & 0,018 & oc \\
\hline Cyanocorax chrysops (Vieillot, 1818) & M.P & 28 & 48 & 0,100 & 0,100 & res \\
\hline \multicolumn{7}{|l|}{ Troglodytidae } \\
\hline Troglodytes aedon Vieillot, 1808 & M.P & 16 & 32 & - & - & res \\
\hline \multicolumn{7}{|l|}{ Mimidae } \\
\hline Mimus saturninus (Lichtenstein, 1823) & $M, P$ & 4 & 20 & - & - & oc \\
\hline Donacobius atricapillus (Linnaeus, 1766 ) & B & 12 & 80 & - & - & oc \\
\hline
\end{tabular}


Tabela III. Continuação.

\begin{tabular}{|c|c|c|c|c|c|c|}
\hline \multirow{2}{*}{ Espécies } & \multirow{2}{*}{ HAB } & \multicolumn{2}{|c|}{ FO $(\%)$} & \multicolumn{2}{|c|}{ IPA } & \multirow{2}{*}{ Status } \\
\hline & & SE & $\mathrm{MC}$ & $\mathrm{SE}$ & MC & \\
\hline \multicolumn{7}{|l|}{ Turdidae } \\
\hline Turdus rufiventris Vieillot, 1818 & M & 12 & 16 & 0,045 & 0,027 & res \\
\hline Turdus leucomelas Vieillot, 1818 & M & 32 & 72 & 0,109 & 0,364 & mig \\
\hline Turdus amaurochalinus Cabanis, 1851 & M & - & 4 & - & - & vag \\
\hline \multicolumn{7}{|l|}{ Vireonidae } \\
\hline Cyclarhis gujanensis (Gmelin, 1789) & M & 92 & 68 & 0,500 & 0,191 & res \\
\hline Vireo olivaceus (Linnaeus, 1766) & M & 72 & 20 & 0,418 & 0,036 & mig \\
\hline Hylophilus poicilotis Temminck, 1822 & M & - & 4 & - & - & vag \\
\hline \multicolumn{7}{|l|}{ Icteridae } \\
\hline Molothrus bonariensis (Gmelin, 1789) & $\mathrm{P}$ & 12 & 4 & - & - & oc \\
\hline Cacicus haemorrhous (Linnaeus, 1766) & M & 4 & 92 & 0,009 & 0,536 & res \\
\hline Gnorimopsar chopi (Vieillot, 1819) & $\mathrm{P}$ & 4 & 4 & - & - & oc \\
\hline Agelaius ruficapillus Vieillot, 1819 & B & - & 16 & - & - & oc \\
\hline Icterus cayanensis (Linnaeus, 1766) & M & 4 & 8 & - & 0,073 & vag \\
\hline Pseudoleistes guirahuro (Vieillot, 1819) & $\mathrm{P}$ & 4 & 4 & - & - & oc \\
\hline \multicolumn{7}{|l|}{ Parulidae } \\
\hline Geothlypis aequinoctialis (Gmelin, 1789) & $M, B$ & - & 8 & - & - & oc \\
\hline Basileuterus flaveolus (Baird, 1865) & M & 96 & 88 & 0,509 & 0,736 & res \\
\hline Basileuterus culicivorus (Lichtenstein, 1830) & M & 88 & 84 & 0,545 & 0,418 & res \\
\hline \multicolumn{7}{|l|}{ Coerebidae } \\
\hline Conirostrum speciosum (Temminck, 1824) & M & 8 & 4 & - & - & vag \\
\hline Dacnis cayana (Linnaeus, 1766) & M & 8 & 4 & - & - & vag \\
\hline \multicolumn{7}{|l|}{ Thraupidae } \\
\hline Euphonia chlorotica (Linnaeus, 1766) & M & 44 & 40 & 0,082 & 0,182 & res \\
\hline Tangara cayana (Linnaeus, 1766) & $\mathrm{P}$ & 8 & 4 & 0,009 & - & vag \\
\hline Thraupis sayaca (Linnaeus, 1766) & $\mathrm{M}, \mathrm{P}$ & 28 & 48 & 0,036 & 0,091 & res \\
\hline Ramphocelus carbo (Pallas, 1764) & M & 48 & 36 & 0,091 & 0,109 & res \\
\hline Habia rubica (Vieillot, 1817) & M & 84 & - & 0,373 & - & res \\
\hline Tachyphonus coronatus (Vieillot, 1822) & M & 20 & 12 & 0,027 & 0,027 & res \\
\hline Nemosia pileata (Boddaert, 1783) & M & 8 & 8 & - & - & vag \\
\hline Thlypopsis sordida (Lafresnaye \& d'Orbigny, 1837) & M & 4 & - & - & - & vag \\
\hline \multicolumn{7}{|l|}{ Fringillidae } \\
\hline Saltator atricollis Vieillot, 1817 & $\mathrm{P}$ & 4 & 8 & - & - & oc \\
\hline Pitylus fuliginosus (Daudin, 1800) & M & 12 & 36 & 0,009 & 0,082 & res \\
\hline Volatinia jacarina (Linnaeus, 1766) & $\mathrm{P}$ & 4 & 56 & - & - & oc \\
\hline Sporophila lineola (Linnaeus, 1758) & M & 4 & - & - & - & vag \\
\hline Sporophila caerulescens (Vieillot, 1817) & M & 4 & 12 & - & 0,036 & vag \\
\hline Coryphospingus cucullatus (Maller, 1776) & M & 8 & 12 & - & - & oc \\
\hline Arremon flavirostris Swainson, 1837 & M & 28 & 20 & 0,036 & 0,018 & res \\
\hline Myiospiza humeralis (Bosc, 1792) & $P$ & - & 12 & - & - & oc \\
\hline Zonotrichia capensis (Müller, 1776) & $M, P$ & 8 & 8 & - & 0,009 & oc \\
\hline
\end{tabular}

O total de espécies encontradas no levantamento quantitativo em 110 amostras (69 em SE e 75 em MC) é inferior ao observado por ALEIXO \& VIELLIARD (1995) com 82 espécies em 202 amostras e Vielliard \& SilVA (1990) com 111 espécies em 110 amostras.

O pequeno tamanho e o grau de isolamento das áreas podem ser os fatores responsáveis pelo baixo número de espécies encontradas no levantamento quantitativo, porém estes resultados são difíceis de serem interpretados devido à escassez de levantamentos quantificados de comunidades de aves em matas ciliares que estabeleçam uma base de comparação. 


\section{Número de contatos}

Para o total de 110 amostras, foram registrados 829 contatos em SE com uma média de 7,5 contatos por amostra e 1107 contatos em MC com uma média de 10,1 contatos por amostra. A soma do número de contatos obtidos num mesmo dia, dividido pelo total de pontos amostrados naquele dia, representa o índice pontual de abundância (IPA global) da avifauna nesta data. A média do IPA de visitas efetuadas em cada mês (IPA médio mensal) variou de 4,6 a 8,2 em Santa Elisa (variação de 78\%) e de 4,2 a 12,2 em Morro Chato (variação de 190\%) (Fig. 2).

A maior conspicuidade das manifestações sonoras das aves nos meses de setembro a novembro pode ter sido responsável pelo maior registro de espécies e maiores IPAs nestas datas como ilustrado nas figuras 1 e 2 .

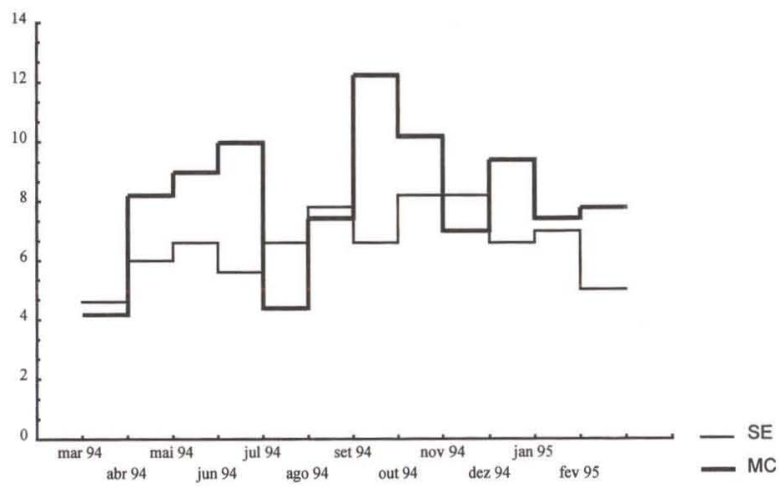

Fig. 2. Variação do índice pontual de abundância em Santa Elisa e Morro Chato.

Índice pontual de abundância (IPA) por espécie

O IPA por espécie amostrada relaciona o número médio de contatos dessa espécie com o número total de amostras. O IPA é um valor relativo, porém comparável somente entre medidas da mesma espécie em datas, locais e comunidades diferentes (ALEIXO \& VIELLIARD 1995). Em Santa Elisa o IPA específico variou entre 0,009 (um contato) e 0,545 (60 contatos), em Morro Chato o IPA variou entre 0,009 (um contato) e 1,082 (119 contatos) (Tab. III).

A ordenação decrescente dos valores do IPA para cada espécie mostra a repartição da abundância relativa das espécies da comunidade. Nota-se através das figuras 3 e 4 que existem em SE e MC poucas espécies com altos valores de IPA e um grande número de espécies com valores intermediários e baixos, conforme 0 padrão observado em outros levantamentos (VIELliaRD \& Silva 1990; AlEIXO \& VIELLIARD 1995). Em SE o valor do IPA mais alto não ultrapassa 0,6 e em MC apenas duas espécies possuem IPA maior que 0,6 (Pitangus sulphuratus e Basileuterus flaveolus). Dentre as espécies com IPA elevado, estão aquelas com canto de longo alcance ou vocalizações constantes como P. sulphuratus, Herpsilochmus pileatus, B. flaveolus, Antilophia galeata (Lichtenstein, 1823) e Habia rubica (Vieillot, 1817). 


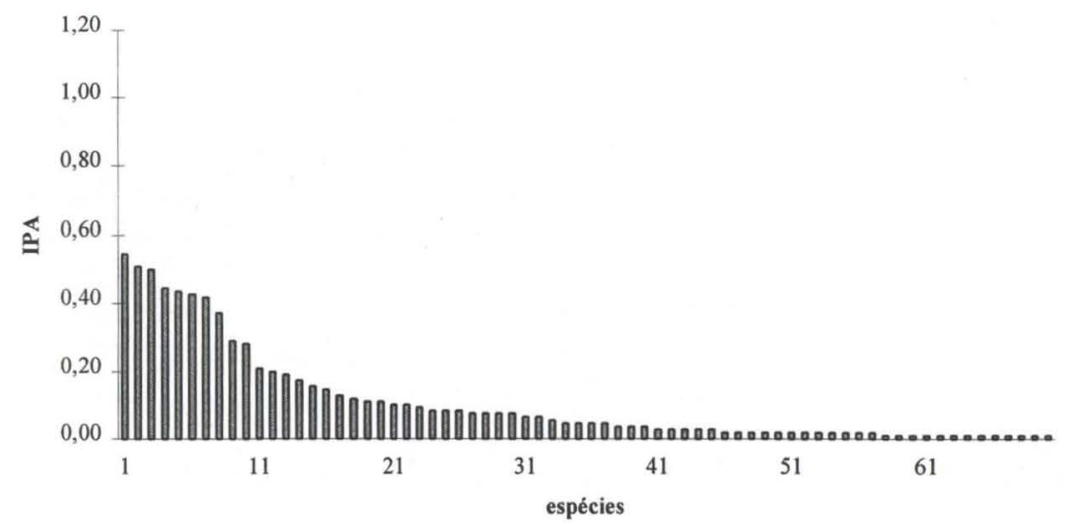

Fig. 3. Distribuição dos índices pontuais de abundância por espécie em ordem decrescente (Santa Elisa).

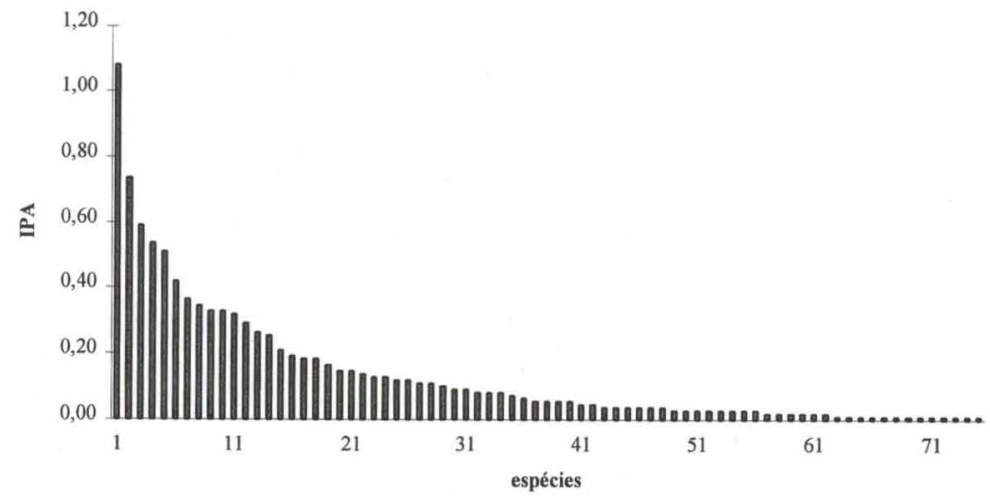

Fig. 4. Distribuição dos indices pontuais de abundância por espécie em ordem decrescente (Morro Chato).

Algumas espécies apresentaram um IPA elevado em uma área e baixo em outra. Este é o caso de Thamnophilus doliatus Linnaeus (1764), P. sulphuratus e Cacicus haemorrhous (Linnaeus, 1766), que tiveram um alto IPA em Morro Chato e um baixo IPA em Santa Elisa. Por outro lado, Conopophaga lineata (Wied, 1831) e Vireo olivaceus (Linnaeus, 1766) apresentaram IPA elevado em SE e baixo em $\mathrm{MC}$.

Outras espécies foram encontradas em uma área apenas, mas com um alto valor de IPA. É o caso de Synallaxis ruficapilla (Vieillot, 1819) e Habia rubica em SE e de Corythopis delalandi (Lesson, 1831) em MC. Uma possível explicação para a ausência de $C$. delalandi em SE e de $S$. ruficapilla e $H$. rubica em MC é a de que, dada sua distribuição não homogênea no ambiente original, seus territórios não foram englobados durante o processo de fragmentação (BIERREGARD et al. 1992). Este fenômeno é chamado de exclusão inicial por MEFFE \& CARROL (1994). A exclusão de espécies em determinadas áreas também pode ocorrer pela não adapta- 
ção ao ambiente simplificado, intensificação da competição interespecífica por recursos que foram limitados, ou fragmentação de sua área de ocorrência. A extinção local também poderia explicar ausências nas áreas estudadas, mas somente estudos a longo prazo sobre a ecologia das espécies poderiam oferecer uma resposta segura a respeito.

\section{Conservação e manejo}

A quantificação e o monitoramento das populações de aves ao longo dos anos pode contribuir para uma melhor compreensão dos efeitos da fragmentação. $\mathrm{O}$ conhecimento da estrutura das comunidades de aves existentes nos fragmentos florestais é um fator importante na elaboração de projetos que visem a recuperação, conservação e manejo das matas ciliares.

Para minimizar os efeitos da fragmentação nesses ambientes, algumas propostas são feitas como: estabelecer um plano de recuperação da bacia com replantio das áreas desmatadas e criação de corredores de vegetação interligando os vários fragmentos da bacia do rio Jacaré-Pepira, realizar programa de monitoramento ambiental destes fragmentos ao longo dos anos, e formar grupos interdisciplinares que tenham como objetivo a recuperação e manutenção destas áreas.

AGRADECIMENTOS. Os autores agradecem ao Programa de Pós-Graduação em Ecologia e Recursos Naturais da UFSCar pelo apoio logístico e ao CNPq pela bolsa concedida. Ao Eng1 Florestal Cássio H. G. Cezare e ao técnico Luiz Aparecido Joaquim (DEBE/UFSCar), pelo acompanhamento nos trabalhos de campo.

\section{REFERÊNCIAS BIBLIOGRÁFICAS}

AleiXo, A. \& J. VielLiard. 1995. Composição e dinâmica da avifauna da mata de Santa Genebra, Campinas, São Paulo, Brasil. Revta bras. Zool. 12 (3): 493-511. Bierregard JR., R.O.; T.E. Lovejoy; V. Kapos; A.A. SAntos \& R.W. HUTCHINGS. 1992. The biological dynamics of tropical rainforest fragments. Bioscience 42 (11): 859-866.

Blondel, J.; C. FERRY \& B. Frochot. 1970. La méthode des indices ponctuels d'abondance (I.P.A.) ou des relevés d'avifaune par "stations d'écoute". Alauda 38: $55-71$.

MefFe, G.F. \& C.R. CARROL. 1994. Habitat fragmentation, p.237-264. In: G.F. MEFFE \& C.R. CARROL (Eds). Principles of conservation biology. Sunderland, Massachusetts, Sinauer Associates Inc., 600p.

Meyer DE SChAuEnSEe, R. 1966. The species of birds of South America and their distribution. Philadelphia, The Academy of Natural Sciences of Philadelphia, XVIII+578p.

- 1970. A guide to the birds of South America. Philadelphia, The Academy of Natural Sciences of Philadelphia, XIV+470p.

Silva, W.R. \& J. Vielliard (no prelo). Avifauna de mata ciliar. In: H.F. LEITÃo-FILHO \& R.R. RODRIGUES (Eds). Matas ciliares: estado atual do conhecimento. Campinas, Editora da Unicamp.

VIELLIARD, J. (no prelo). Birds community as an indicator of biodiversity: results 
from quantitative surveys in Brazil. Anais da Academia Brasileira de Ciências.

Vielliard, J. \& W.R. Silva. 1990. Nova metodologia de levantamento quantitativo da avifauna e primeiros resultados no interior do Estado de São Paulo, Brasil. Anais do IV ENAV, Recife, Universidade Federal Rural de Pernambuco, p. 117-151

Recebido em 11.XII.1997; aceito em 07.X.1999. 